\title{
Chronic Familial Mediterranean Fever with Development of Secondary Amyloidosis
}

\section{CHRISTOPHER LARRIMORE}

\begin{abstract}
A 20-year-old Turkish male presented with fever, abdominal pain, and systemic lethargy. His clinical history revealed symptoms to be self-limiting but reoccurring over the past six months. Blood and urine specimens collected indicated renal amyloidosis. A kidney CT image indicated kidney inflammation. He was diagnosed with Familial Mediterranean Fever with the development of secondary amyloidosis and treated with colchicine.
\end{abstract}

ABBREVIATIONS: CT = computerized tomography; FMF $=$ Familial Mediterranean Fever; $\mathrm{WBC}=$ white blood cell count; RBC = red blood cell count; BUN = blood urea nitrogen; eGFR = estimated glomerular filtration rate; MEFV = Mediterranean Fever Gene; $\mathrm{PCR}=$ polymerase chain reaction

INDEX TERMS: Familial Mediterranean Fever; Secondary Amyloidosis; Colchicine; Autosomal Recessive Disorders; Mediterranean Fever Gene

Clin Lab Sci 2011;24(1):2

Christopher Larrimore, North Annapolis, MD 21409

Address for correspondence: Christopher Larrimore, 1006 Commander's Way North Annapolis, MD 21409, (410)

725-6609,cwlarrimore@hotmail.com

\section{CASE HISTORY}

A 20-year-old male of Turkish descent presented to his family physician with a fever of $101.9^{\circ} \mathrm{F}$, abdominal discomfort, and systemic lethargy. Discussion with the patient revealed that the onset of his symptoms began approximately six months earlier. The patient had been experiencing reoccurring episodes of fever with the gradual increase of abdominal pain during each subsequent episode. These episodes were self-limiting usually lasting between 24 to 72 hours, followed by prolonged non-symptomatic periods that demonstrated no observable patterns. Additionally, the patient revealed that two of his male relatives also complained of similar reoccurring fevers, however without abdominal pain or systemic lethargy. A clinical examination of the patient revealed severe tenderness when pressure was applied to the right upper quadrant of the abdominopelvic region. Due to the severity of the patient's complaints and his relevant clinical history, the patient was admitted to a local hospital where blood and urine specimens were collected and a computerized tomography (CT) scan of the abdomen was performed.

The CT generated image indicated the presence of moderate inflammation of the right kidney. His blood and urine results came back abnormal and demonstrated a heightened immune response with acute renal failure; demonstrated by tables 1 and 2. Based upon the laboratory results and the patient's clinical and family history, Chronic Familial Mediterranean Fever (FMF) was suspected. Subsequently, a polymerase chain reaction (PCR) test was performed to identify the presence of any known FMF gene mutations. The results came back positive and the patient was diagnosed with FMF with the development of secondary amyloidosis of the kidney. The patient was started on a regimen of $0.6 \mathrm{mg}$ of colchicine taken three times a day. After the first 24 hours of treatment symptoms began to subside. Within three days, additional laboratory tests showed improved kidney function and decreased immune response. The patient was discharged from the hospital after three days.

\section{BACKGROUND}

Familial Mediterranean Fever (FMF), also known as Armenian disease or paroxysmal polyserositis is a hereditary inflammatory disease that is commonly 


\section{CLINICAL PRACTICE}

inherited among people of Mediterranean origin. ${ }^{1}$ FMF is characterized by recurrent attacks of fever that can be accompanied by self-limiting arthritis, pleuritis, peritonitis and the possible development of secondary amyloidosis. Approximately one out of every two hundred people among the Jewish, Armenian, Arab, and Turkish populations have inherited this autosomal recessive gene disorder with as few as one in five considered carriers. ${ }^{1}$ The disease generally begins in childhood and often continues intermittently throughout life. Epidemiology studies reveal that the most common ages for symptoms to begin are between years $18-20 .^{2,3}$ and males are more often affected than females. Furthermore, although the occurrences of symptoms are difficult to predict, and the secondary complications associated with the disease can be very severe, once patients are treated with prescription medication the disease becomes easily manageable. ${ }^{2,3}$

Typical symptoms that are present in FMF patients include irregular occurring attacks of fever lasting between 24 to 72 hours, polyserositis causing severe abdominal or pleuritic pain, attacks of arthritis that usually last up to 7 days and possibly painful, swollen skin lesion on the lower legs. ${ }^{1}$ The severity and frequency of these symptoms are unpredictable, they can occur as often as twice a week or as few as once a year. Often a major complication of FMF is the development of secondary amyloidosis, this usually affects the kidneys. ${ }^{4}$ Symptoms also associated with renal amyloidosis include weakness, fatigue, and swelling of the kidneys. ${ }^{4}$ The prognosis of FMF with the development of secondary amyloidosis is often good but depends on the severity of any damage resulting from amyloidosis and if treatment has been appropriately provided.

The inherited gene that is responsible for FMF is the Mediterranean Fever Gene (MEFV). This gene is located on the short arm of chromosome 16 at position 13.3 between nucleotide base pairs 3,232,028 and base pairs 3,246,627., 5 This gene is composed of 10 exons and extends a length of approximately $14 \mathrm{~Kb}$ of the genomic DNA (5). MEFV provides instructions for the cellular production of a protein named pyrin, also known as marenostrin. ${ }^{5,6}$ When nonsense or missense mutations in the MEFV gene occur, pyrin becomes either mutated or not produced. Pyrin is an important protein that is produced in white blood cells (primarily neutrophils, eosinophils and monocytes) and although the entire function of pyrin is not completely yet known, it is thought to play a major role in the regulation of inflammation and infection.

Some of the evidence that implicates pyrin as a regulator of inflammation includes its interaction with leukocyte cytoskeleton, the death domain contained within the protein and its ability to deregulate chemotactic factors. Pyrin has been observed to bind to the cytoskeleton of leukocytes and although not much information is known about this interaction, if the cytoskeleton is responsible for defining a cell's shape, size and movement, then this interaction may influence the cell's ability to regulate inflammation or infection. ${ }^{6}$ The protein also contains a death domain called PYD (Pyrin Domain). ${ }^{5}$ This domain has been widely associated with other proteins that are actively involved in apoptosis. ${ }^{7}$ The presence of this domain suggests that pyrin may also have an influential role in cell apoptosis. ${ }^{5}$ Lastly, pyrin has been associated with the deregulation of chemotactic factor C5a and interleukin-8., ${ }^{5,9}$ It has been observed that while patients with normal pyrin have the ability to deregulate mediators of an inflammatory response, such as C5a and interleukin-8, patients with abnormal pyrin tend to develop a large accumulation of C5a and IL-8. Overall, the chronic inflammation seen in FMF patients, which is likely due to the abnormal pyrin, leads to the accumulation of acute phase proteins and deposit of acute phase protein amyloid $\mathrm{A}$ into various tissues. ${ }^{10}$ If this accumulation persists, then the outcome will be an interference of normal tissue structure and function; this type of disturbance is classified as amyloidosis. ${ }^{10}$

Amyloidosis is a rare and potentially fatal disease that can be either localized or systemic. ${ }^{4}$ There are four major types of amyloidosis. The four major types include immunoglobulin (primary) amyloidosis, reactive (secondary) amyloidosis, beta- 2 microglobulin amyloidosis and hereditary amyloidosis. Each different type of amyloidosis presents a different prognosis and stems from different underlining conditions. The pathologic features of amyloid deposits include betapleated sheet structures that are composed of amyloid 


\section{CLINICAL PRACTICE}

fibrils with diameters between 8 to $10 \mathrm{~nm}^{10}$ Betapleated sheets can be viewed under polarized light after being stained using Congo Red stain, these stained fibrils display an apple green birefringence. ${ }^{10}$

Secondary amyloidosis is associated with chronic inflammatory diseases such as FMF. ${ }^{4,10}$ The precursor protein responsible for constructing the amyloid fibrils associated with secondary amyloidosis is serum amyloid A, an acute-phase reactant. ${ }^{10}$ Typical sites of amyloid accumulation include the spleen, liver, lymph nodes, adrenal glands, and the kidneys. ${ }^{4}$ Symptoms that are nonspecific include complaints of weakness and fatigue. Specific complaints are directly associated to organ involvement, these symptoms commonly include edema and pain. While secondary amyloidosis can lead to organ dysfunction and failure, it can be treated using prescription colchicine and if treated early enough, the organ damage can possibly be reversed. ${ }^{8}$

Episodes of fever are also linked to the absence of pyrin. Fever is induced by pyrogens that can either be endogenous (cytokines) or exogenous (bacteria). Pyrogens cause the release of prostaglandin E2 (PGE2), PGE2 acts upon the hypothalamus and results in the increase of body temperature. Without adequate regulation of inflammation or infection by pyrin, overproduction of cytokines related to inducing fever (interleukin-1, interleukin-6, interleukin-8 and tumor necrosis factor alpha) increase in concentration. ${ }^{11}$ Increased concentrations of these fever-inducing cytokines results from both increased concentration of white blood cells and increased activity of white blood cells in response to inflammation or infection.

\section{CORRELATION OF LABORATORY RESULTS}

Most standard laboratory tests are non-informative, however results that have correlation to FMF during non-symptomatic periods include elevated erythrocyte sedimentation rate and elevated concentrations of white blood cells and polymorphonuclear leukocytes. ${ }^{5}$ During attacks or immediately following an attack, laboratory results will show decreased albumin concentrations, deficient complement C5aINH (an inhibitor of the innate immune system's complement cascade), elevated levels of fibrinogen, C-reactive protein, amyloid A protein and haptoglobin. ${ }^{5}$ Studies measuring immunoglobulin (Ig) levels in the sera found that $\operatorname{IgA}$ increased 23\%, IgM increased 13\%, IgG increased 17\% and $\mathrm{IgD}$ increased $13 \%$. Since FMF is due to the presence of the MEFV gene, PCR can be used to identify some of the more frequent mutations, these mutations are listed in Table 3., ${ }^{5,6}$ And although there are many different mutations possible, mutations M6994V and V726A have the most correlation to FMF with renal amyloidosis. ${ }^{5,6}$ Once FMF was suspected, the patient was tested for mutations M6994V and V726A. Mutation V726A was resulted as being present.

Indicated by Table 1 are the patient's hematology and chemistry results that clearly indicate a heightened immune response. The elevated white blood cell count, elevated erythrocyte sedimentation rate, increased presence of band neutrophils, elevated concentrations of neutrophils and the increased concentration of acute phase proteins demonstrate this immune response. Acute phase proteins increase at the onset of an inflammatory response, due to the patient's chronic fever, acute phase proteins and a progressive shift in white blood cell type has occurred. ${ }^{11}$ Chronic fever causes an increased production of white blood cells. Neutrophils, an essential part of the innate immune system, are the most abundant white blood cell type in the body and function primarily to fight infection and inflammation. ${ }^{11}$ In FMF there exists a mutation in the pyrin gene of neutrophils which leads to a constitutively active acute phase response followed by increased production of neutrophils and an increased acute phase protein response. ${ }^{6,12}$

Acute phase proteins are present in the blood and increase in concentration when inflammation occurs. There are several different classes of acute phase proteins that each performs different physiological functions for the immune system. Two of the most prominent proteins are $\mathrm{C}$ - reactive protein and serum amyloid $\mathrm{A}$. In particular, serum amyloid A functions to recruit immune cells to the site of inflammation and induce enzymes to degrade extra cellular matrixes. ${ }^{11}$ As a result to the absence of pyrin, amyloid protein is produced in high concentrations and has accumulated in the kidney and caused inflammation. This accumulation is responsible for the acute kidney failure that is demonstrated by the elevated levels of creatinine and 


\section{CLINICAL PRACTICE}

Table 1. Hematology and Chemistry Laboratory Results

\begin{tabular}{|c|c|c|c|c|c|}
\hline $\begin{array}{l}\text { Analyte } \\
\text { Hematology: }\end{array}$ & ${ }^{1} \mathrm{Adm}$ & Day 1 & Day 2 & Day 3 & ${ }^{2}$ Ref Range \\
\hline Band Neutrophils & 8 & 9 & 7 & 5 & $2-6 \%$ \\
\hline Neutrophils & 75 & 74 & 72 & 69 & $50-70 \%$ \\
\hline Lymphocytes & 10 & 12 & 14 & 19 & $20-44 \%$ \\
\hline Monocytes & 3 & 3 & 4 & 5 & $2-9 \%$ \\
\hline Eosinophils & 4 & 2 & 3 & 2 & $0-4 \%$ \\
\hline Basophils & 0 & 0 & 0 & 0 & $0-2 \%$ \\
\hline RBC count & 3.8 & 4.0 & 4.5 & 4.9 & $4.7-6.1 \times 10^{12} / \mathrm{L}$ \\
\hline Reticulocytes & 2.5 & 2.4 & 1.9 & 1.4 & $0.5-2.0 \%$ \\
\hline Platelets & 215 & 225 & 219 & 223 & $150-400 \times 10^{9} / \mathrm{L}$ \\
\hline${ }^{3}$ Sed Rate & 19 & 17 & 14 & 13 & $0-15 \mathrm{~mm} / \mathrm{hr}$ \\
\hline WBC count & 12.5 & 12.3 & 11.9 & 11.0 & $4.8-10.8 \times 10^{9} / \mathrm{L}$ \\
\hline Hematocrit & 39 & 39 & 40 & 42 & $42-52 \%$ \\
\hline Hemoglobin & 13 & 13 & 13 & 14 & $14-18 \mathrm{~g} / \mathrm{dL}$ \\
\hline \multicolumn{6}{|l|}{ Chemistry: } \\
\hline Creatinine & 1.3 & 1.2 & 1.0 & 1.0 & $0.6-1.2 \mathrm{mg} / \mathrm{dL}$ \\
\hline BUN & 10 & 11 & 13 & 12 & $8-21 \mathrm{mg} / \mathrm{dL}$ \\
\hline C-Reactive Protein & 250 & 100 & 20 & 0.7 & $0.5 \mathrm{mg} / \mathrm{dL}$ \\
\hline Serum Amyloid-A & 25.3 & 13 & 4 & 0.9 & $0.03 \mathrm{mg} / \mathrm{dL}$ \\
\hline eGFR & 28 & 27 & 45 & 59 & $>60 \mathrm{ml} / \mathrm{min}$ \\
\hline
\end{tabular}

${ }^{1} \mathrm{Adm}=$ Admission,${ }^{2}$ Ref $=$ Reference, ${ }^{3}$ Sed $=$ Sedimentation

the low eGFR. ${ }^{13}$

Urine results in Table 2 demonstrate elevated levels of urobilinogen, the abnormal presence of ketones, microalbumin and proteins, and an increased specific gravity. Urobilinogen in the urine suggests hemolytic anemia, this excessive red blood cell breakdown is likely due to the patient's chronic inflammation. ${ }^{13}$ Ketones in the urine are in response to the patient's high fever. High fever increases the body's metabolism and thus causes fat to be utilized to produce energy releasing ketones into the urine. ${ }^{13}$ Microalbuminuria results from the loss of glomerular function resulting in albumin present in the urine. ${ }^{13}$ This occurs due to the damage of tiny blood vessels in the kidneys. Albumin is a protein found in the blood and its presence in the urine is related to acute renal failure. ${ }^{13}$ Like albumin, other proteins should also not be found in the urine, however during renal amyloidosis, amyloid proteins accumulate in the kidney causing proteinuria.

\section{TREATMENT}

Before treatment can begin, there are a few factors that must first be taken into account. These factors include a proper patient diagnosis, a review of symptom occurrences and the evaluation of the patient's response to colchicine. Primarily, a diagnosis of FMF is made if the patient demonstrates associated clinical symptoms, if the patient has an ancestral history of Mediterranean origin, and if genetic test results are positive for MEFV. ${ }^{2,4,8}$ To date there have been more than 35 gene mutations identified as responsible for causing FMF. ${ }^{4}$ Using PCR technology, these known gene mutations can be identified (Table 3 identifies the 16 most clinically relevant mutations). After a diagnosis is made and if the patient is found to be tolerant to colchicine, treatment using colchicine can begin. ${ }^{8}$ 


\section{CLINICAL PRACTICE}

Table 2. Urine and PCR Laboratory Results

\begin{tabular}{lccccc}
\hline Analyte & ${ }^{1}$ Adm & Day $\mathbf{1}$ & Day 2 & Day 3 & ${ }^{2}$ Ref Range \\
Urine: & & & & & \\
Urine RBC & 2 & 1 & 0 & 0 & $0-5 / h p f$ \\
Urine Leukocyte & $\mathrm{Neg}$ & $\mathrm{Neg}$ & $\mathrm{Neg}$ & $\mathrm{Neg}$ & $\mathrm{Neg}$ \\
Urobilinogen & 2.2 & 2.0 & 1.3 & 0.8 & $0.1-1.0$ E.U./dL \\
Urine Bilirubin & $\mathrm{Neg}$ & $\mathrm{Neg}$ & $\mathrm{Neg}$ & $\mathrm{Neg}$ & $\mathrm{Neg}$ \\
Specific Gravity & 1.030 & 1.026 & 1.010 & 1.009 & $1.005-1.030$ \\
pH & 7.10 & 7.13 & 7.25 & 7.26 & $5.0-8.0$ \\
Glucose & $\mathrm{Neg}$ & $\mathrm{Neg}$ & $\mathrm{Neg}$ & $\mathrm{Neg}$ & $\mathrm{Neg}$ \\
Protein & +2 & +2 & $\mathrm{Neg}$ & $\mathrm{Neg}$ & $\mathrm{Neg}$ \\
Ketones & Trace & $\mathrm{Neg}$ & $\mathrm{Neg}$ & $\mathrm{Neg}$ & $\mathrm{Neg}$ \\
Microalbumin & Present & Present & $\mathrm{Neg}$ & $\mathrm{Neg}$ & $\mathrm{Neg}$ \\
PCR: & & & & & \\
Mutations & $\mathrm{V}$ & & & & \\
\hline
\end{tabular}

${ }^{1} \mathrm{Adm}=$ Admission, ${ }^{2} \mathrm{Ref}=$ Reference

Table 3. Clinically Relevant Mutations of MEFV

\begin{tabular}{cl}
\hline Exon & \multicolumn{1}{c}{ Mutation } \\
2 & E148Q, E167D, T267I \\
3 & P369S \\
5 & R408Q, F479L \\
10 & M680I (x2), T681I, 1692del, M694del, M694V, \\
& M694I, K695R, V726A, A744S, R761H
\end{tabular}

Colchicine is a FDA approved drug that is commonly prescribed to treat diseases such as FMF, gout and Behcet's disease. ${ }^{14}$ The molecule's biological functions include microtubule polymerization and actin polymerization. ${ }^{15}$ Colchicine polymerizes microtubules by binding to beta-tubulins, a major constituent of microtubules. When the tubulins become functionally unavailable due to the binding of cochicine (betatubulin-colchicine complex) mitosis becomes inhibited and cell motility and activity become altered. ${ }^{15}$ Actin polymerization that is generated by colchicine results in neutrophil cell rigidity. ${ }^{15}$ Together these two forms of polymerization (microtubule and actin) lead to an antiinflammatory effect that reduces the inflammation that is observed in FMF patients. If colchicine treatment is provided within reasonable time, the accumulation of amyloid protein in the tissue can be impeded and secondary amyloidosis prevented or reversed (dependent on the severity).

Colchicine provides the most effective treatment for many FMF patients. This medication should be taken daily to decrease episode frequency and severity and to prevent or reverse secondary amyloidosis that has developed. It primarily functions by decreasing the motility of leukocytes and decreasing phagocytosis in inflammatory responses. Colchicine is relatively safe with few noted side effects that consist of gastrointestinal upset and neutropenia. The effective dosage reported by Livneh et al in a study of 68 FMF patients who also had complications of secondary renal amyloidosis is $>1.5 \mathrm{mg} /$ day; however typical dosage prescribed range from $0.5-2 \mathrm{mg} /$ day. ${ }^{16}$ Other possible treatment options include analgesia or non-steroidal anti-inflammatory drugs like diclofenac, etodolac or sulindac. ${ }^{17}$ Corticosteroids have demonstrated not to be greatly effective and in some cases they have shown to be ineffective, the explanation for corticosteroid ineffectiveness with FMF treatment is still not fully understood. $^{2}$ 


\section{CLINICAL PRACTICE}

\section{CONCLUSION}

When the patient was discharged from the hospital he had successfully been treated for FMF with the development of secondary amyloidosis of his right kidney. Laboratory results provided supportive evidence of inflammation and kidney failure. The key findings that lead to the diagnosis of FMF were the discovery of an MEFV mutation using PCR combined with the patient's history. The patient was given a total daily dosage of $1.8 \mathrm{mg}$ of colchicine and monitored for improvement. As the inflammation decreased so did the accumulation of amyloid in the kidneys, and function was restored. Since this disease is a genetic disorder, the patient will likely continue to have FMF episodes. However, with daily usage of colchicine the frequency and severity should be dramatically reduced.

\section{REFEERENCES}

1. National Human Genome Research Institute: Learning About Familial Mediterranean Fever. Available from http://www. genome.gov/12510679. Accessed 2010 Jun 25.

2. Thoene J, Doris S, editors. Physician's Guide to Rare Diseases. New Jersey: Dowden Publishing Company; 1992.

3. Warrell D, Cox T, editors. Oxford Textbook of Medicine. $4^{\text {th }}$ ed. Oxford University Press: 2005.

4. LeBlond R, Brown D, DeGowin R, editors. De Gowin's Diagnostic Examination. 9th ed. New York: McGraw-Hill; 2009.

5. Medlej-Hashim M, Loiselet J, Lefranc G, Megarbane A. Familial Mediterranean Fever (FMF): from diagnosis to treatment. Sante. 2004; 14(4):261-6.
6. Genetics Home Reference: MEFV. Available from http://ghr.nlm.nih.gov/gene=mefv. Accessed 2010 Jun 25.

7. Reed J, editor. Comparative analysis of apoptosis and inflammation genes of mice and humans. Genome Research. 2003; 13(6B):1376-88

8. McPhee S, Papadakis M, Tierng L, editors. Current Medical Diagnosis \& Treatment. 47th ed. New York, Chicago, San Francisco: McGraw-Hill; 2008.

9. Wallach J, editor. Interpretation of Diagnostic Tests: A Synopsis of Laboratory Medicine. 5th ed. Boston: Little, Brown and Company; 1992.

10. Koopman W, editor. Clinical Primer Of Rheumatology. Philadelphia: Lippincott Williams \& Wilkins; 2003.

11. Stevens C, editor. Clinical Immunology and Serology. 2nd ed. Philadelphia: F.A. Davis Company; 2003.

12. Genetics Home Reference: Familial Mediterranean Fever. Available from http://ghr.nlm.nih.gov/condition=familial mediterraneanfever. Accessed 2010 Jun 25.

13. Strasinger S, editor. Urinalysis And Body Fluids. 3rd ed. Philadelphia: F.A. Davis Company; 1989.

14. U.S. Food and Drug Administration: News and Events. Available from http://www.fda.gov/NewsEvents/Newsroom/ PressAnnouncements/ucm 174620.htm. Accessed 2010 Jun 25.

15. Tsai MA, Waugh RE, Keng PC. Passive mechanical behavior of human neutrophils: effects of colchicine and paclitaxel. Biophysical Journal. 1998; 74(6):3282-91.

16. Livneh A, Zemer D, Langevitz P, Laor A, Sohar E, Pras M. Colchicine treatment of AA amyloidosis of familial Mediterranean fever. An analysis of factors affecting outcome. Arthritis Rheum. 1994; 37(12):1804-11.

17. Merck: Amyloidosis. Available from http://www.merck.com/ mmhe/sec25/ch304/ch304a.html. Accessed 2010 Jun 25.

The peer-reviewed Clinical Practice Section seeks to publish case studies, reports, and articles that are immediately useful, are of a practical nature, or contain information that could lead to improvement in the quality of the clinical laboratory's contribution to patient care, including brief reviews of books, computer programs, audiovisual materials, or other materials of interest to readers. Direct all inquiries to Elizabeth Kenimer Leibach, EdD MS MLS SBB,Departments of Biomedical and Radiological Technologies and Pathology, Medical College of Georgia, EC 2437, Augusta, GA 30912

Clinical Laboratory Science encourages readers to respond with thoughts, questions, or comments regarding these articles. Email responses to westminsterpublishers@comcast.net. In the subject line, please type the journal issue and lead author such as "CLIN LAB SCI 24(1) RE LARRIMORE". Selected responses may appear in the Dialogue and Discussion section in a future issue. Responses may be edited for length and clarity. We look forward to hearing from you. 